\title{
Simulation of the Time Evolution Profiles in Nanoimprint Lithography
}

\author{
Toshiaki Tanabe, Masaaki Nishihata, Hiroyuki Kawata, and Yoshihiko Hirai
}

\author{
Department of Physics and Electronics Engineering, \\ Osaka Prefecture University \\ 1-1 Gakuen-cho, Sakai, 599-8531, Osaka, Japan
}

\begin{abstract}
To study the time dependence of the resist profiles in nanoimprint lithography, numerical simulation has been done by using conventional finite element method, where the resist is assumed as a viscous fluid body over the glass transition temperature. On the other hand, the resist shape is quenched by rapid cooling just after pressing the resist. Time dependent resist profiles are experimentally observed for various pressing times. The resist filling rate into the mold cavity is evaluated and they relatively agree with each other qualitatively. Also, the dependence of the time evolutions on the aspect ratio of the mold groove is simulated and there exist minimum aspect ratio to shorten the process time.
\end{abstract}

Keywords: nanoimprint, resist, process time, time dependence, time evolution

\section{Introduction}

Fine pattern transfer technology is strongly demanded for application of various progressing fields such as micro-nano optics devices, nano bio-chips and other advanced devices. Low cost and rapid fabrication technology is indispensable for industrial application. Nanoimprint lithography [1] is one of the promising solutions for nano fabrications, however the process turn around time has not been investigated in details. Time evolution of the deformation process in thermal nanoimprint lithography is important to design the process sequence and optimize the process conditions such as process time and material properties. Several reports have been published but the time dependent resist profiles are not discussed in both theoretically and experimentally [2]-[3].

In this paper, we firstly report the time evolution of the resist profiles in thermal nanoimprint by simplified numerical simulation and experiments.

\section{Numerical simulation}

\subsection{Numerical model}

In the thermal nanoimprint lithography, the thermoplastic polymer is heated over glass transition temperature $(\mathrm{Tg})$ and pressed by fine mold. The polymers over the $\mathrm{Tg}$ is generally characterized by visco-elestic body, which is mechanically characterized combination of two elements by the spring (elastic) and the dashpot (viscous) as shown in Fig. 1. The spring, which is characterized by the storage shear modulus $\mathrm{G}^{*}$, and the dashpot, which is characterized by the loss modulus $\mathrm{G}^{*}$ are connected sequentially as expressed by Maxwell model. Basically, the elastic term $\mathrm{G}^{*}$ is related to the static state and the viscous term dominates transit state in deformation process. These parameters have generally dependency on sheer velocity in the polymer materials and it becomes very complicated to perform precious modeling in numerical simulation.

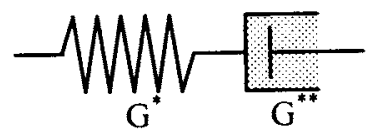

Figure 1. Schematic diagram of the typical mechanical model of the resist polymer over the Tg.

To obtain the resist profiles in static state after long pressing time, we demonstrated resist deformation simulation using rubber elastic model, which ignore the loss modulus (viscous) term [4]. The results fairly simulate the resist profiles after 
imprinting.

In this paper, we neglect the loss modulus (static term) to obtain time evolutions of the resist profiles in the imprinting process to simplify the numerical simulations. To calculate the large deformation process in nanoimprint lithography, we use the MSC-marc system under assumption that the resist property is expressed by the equation (1):

$$
\sigma_{i j}^{\prime}=2 \mu \dot{\varepsilon}_{i j}
$$

, where $\mu$ is the viscosity of the resist at the processing temperature which is derived by experimental measurement of PMMA [5]. The elasto-plastic fluid element is utilized to calculate time evolution of the resist profiles.

\subsection{Analysis model}

The analysis system and boundary conditions are shown in Fig.2. The resist and mold are symmetrical shape and the resist is fixed to the substrate. Contact boundary condition is applied to the resist and mold interface.

The resist is $500 \mathrm{~nm}$ thick PMMA and the mold is rigid body, which has $250 \mathrm{~nm}$ deep lines. The viscosity of the PMMA $\left(\mathrm{M}_{\mathrm{w}}=350 \mathrm{k}\right)$ is $2.77 \times$ $107 \mathrm{~Pa} \cdot \mathrm{s}$ at 140 centigrade. The mold is pressed to the resist under constant pressure (5.0 MPa).

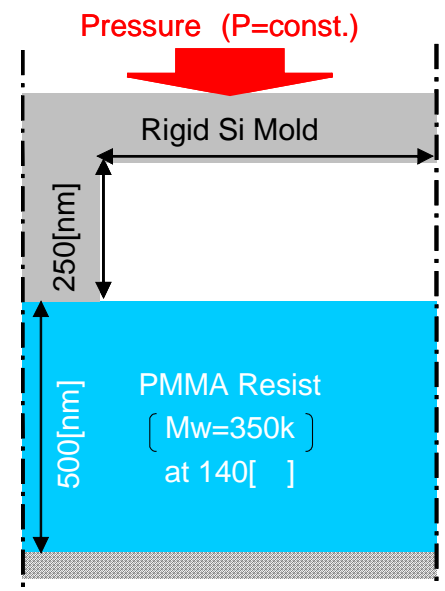

Figure 2. Schematic diagram of the analysis system.

\section{Experiment}

The most important issue to investigate the time evolution of the resist profiles is how to quench the profile in the in-situ process because the resist profile is alternatively modified as the imprint process time proceeds.
In the conventional thermal nanoimprint process, the cooling period takes several minutes to cool down below $\mathrm{Tg}$ because the thermal stage usually has large heat capacity to stabilize the process temperature and it is not easy to cool down below $\mathrm{Tg}$ in short time even by water cooling. The resist profile must be modulated during conventional cooling period.
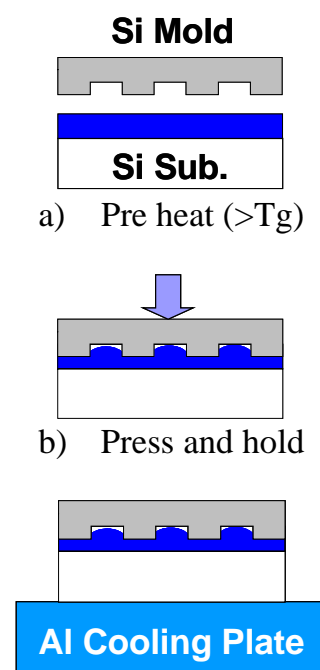

c) Put on the cooling plate

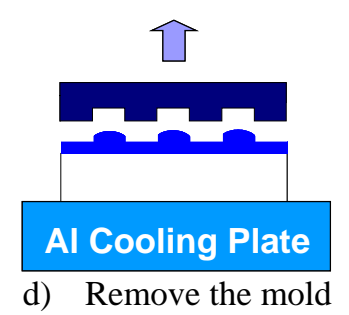

Figure 3. Schematic diagram of the nanoimprint process to quench the resist profile during thermal deformation.

To avoid extra deformation during the cooling process, we newly proposed simple and convenient process as shown in Fig.3.

After heating the resist, the mold is pressed to the resist and hold for the process period. Then, the sample with the mold is removed from the heating stage and rapidly put on the thermal controlled cooling plate within 1 second by hand. We use silicon substrate, which has large thermal conductivity and rapidly cooled down below the Tg. Finally, the mold is removed after quenched the resist profile at low temperature. 


\section{Results and discussions}

\subsection{Time evolutions of the resist profiles}

Figure 4 shows the time evolutions of the resist profiles in both simulation and experiment. The deformation process times are different from each other. This is because the elastic component is ignored in numerical simulation, however the time evolution of the resist profiles relatively agree with each other.

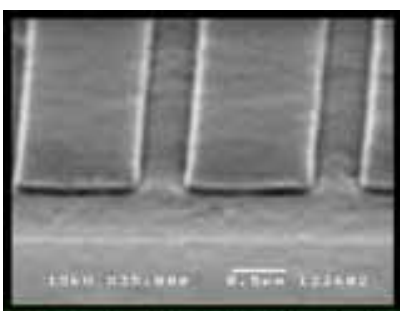

(a) $60 \mathrm{~s}$

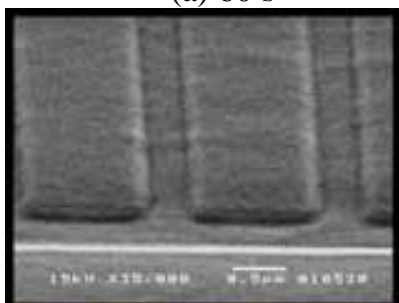

(b) $120 \mathrm{~s}$

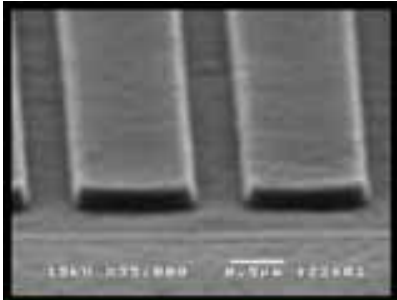

(c) $300 \mathrm{~s}$

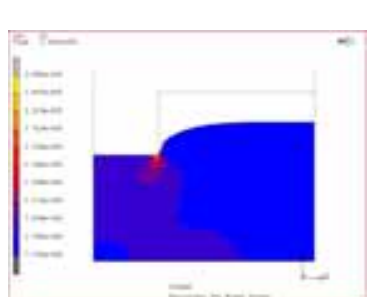

(d) $4 \mathrm{~s}$

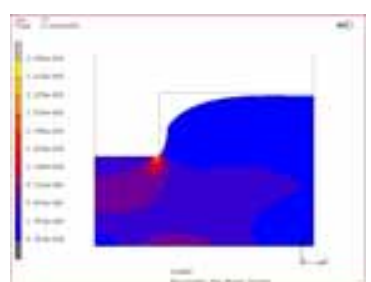

(e) $8 \mathrm{~s}$

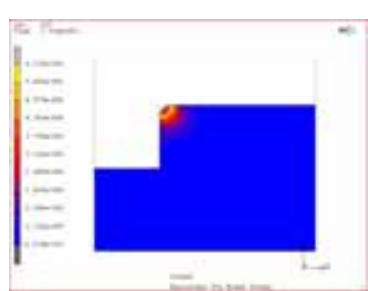

(f) $20 \mathrm{~s}$
Figure 4. Time evolutions of the resist profile for 1200 nm width lines. (Aspect ratio $=0.21$ )

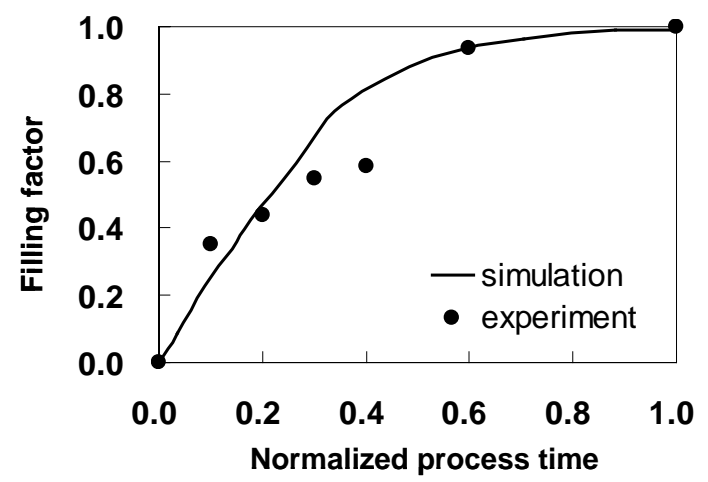

Figure 5. Time evolution of the resist filling rate into the mold cavity. (1200 nm lines)
For quantitative analysis, the resist filling ratio into the mold groove during imprinting is investigated. The filling ratio is defined by volume ratio of the mold cavity vs. deformed resist into the groove, which is approximately derived by the resist height. Figure 5 shows the time evolution of the filling rate in terms of normalized process time, which is derived by dividing the process time by the required time to fill up the mold groove.

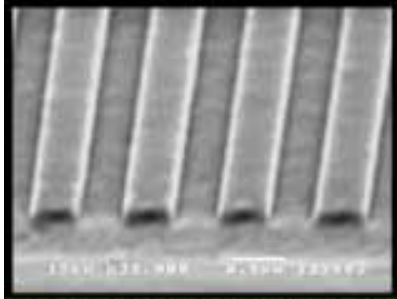

(a) $60 \mathrm{~s}$

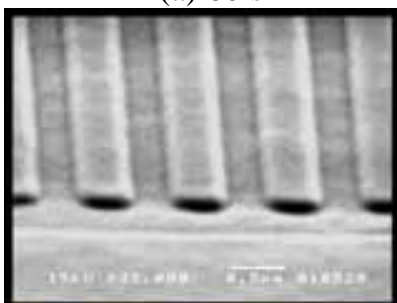

(b) $120 \mathrm{~s}$

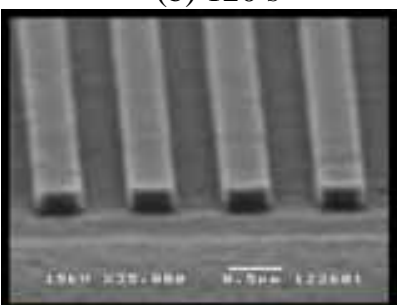

(c) $300 \mathrm{~s}$

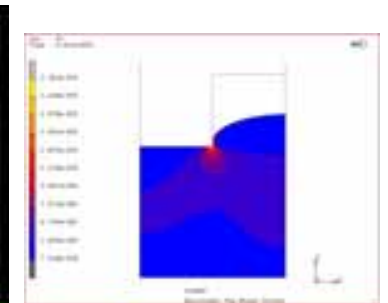

(d) $4 \mathrm{~s}$

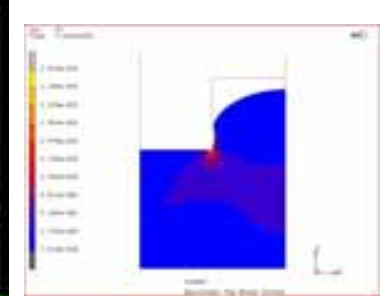

(e) $8 \mathrm{~s}$

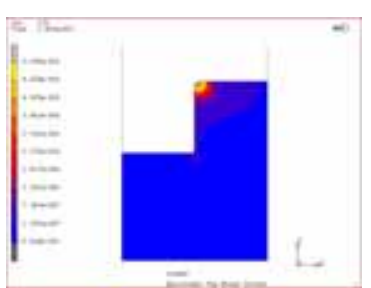

(f) $20 \mathrm{~s}$
Figure 6. Time evolutions of the resist profile for 500 $\mathrm{nm}$ width lines. (Aspect ratio $=0.5$ )

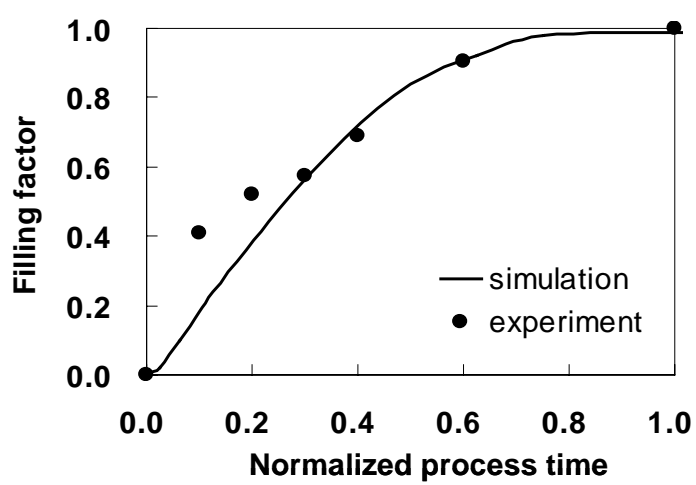

Figure 7. Time evolution of the resist filling rate into the mold cavity. (500 nm lines) 
The simulation and the experiment qualitatively agree with each other. At the beginning of the deformation, the resist is quickly lifted up and then gradually deformed as the time proceeds. The lift up phenomena is dominated by the elastic deformation and the contentious modulation is caused by viscous behavior of the resist. In this simulation, the elastic compornent is neglected and shows rapid deformation.

Figures 6 and 7 show the results for narrow grooves. The line width is around $500 \mathrm{~nm}$. In this case, the required process time is almost the same as the former case (line width $=1200 \mathrm{~nm}$ ). The deformation mechanism is almost the same as discussed above, however it seems to be slow to fill the narrow grooves because the resist is hard to be flow.

\subsection{Dependence on the aspect ratio}

Next, we simulate the time dependency on the aspect ratio by simulation study. Figure 8 shows the time evolutions of the filling factor in various aspect ratio of the mold groove. In this case, the width of the groove is modulated.

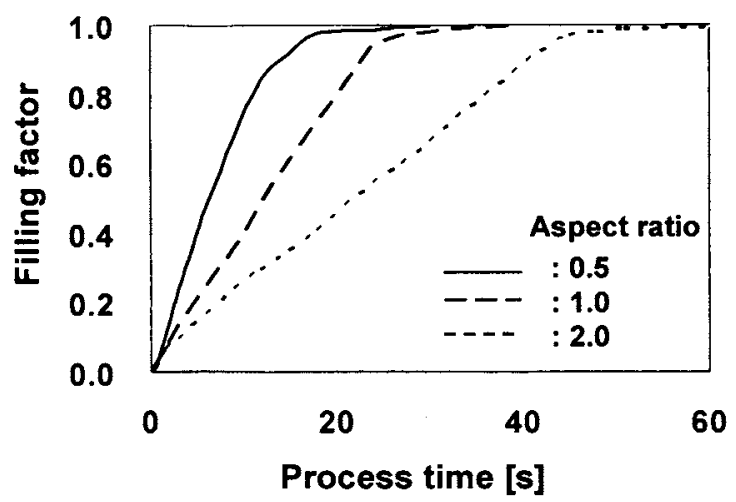

Figure 8. Time evolutions of the filling factor in various aspect ratio of the groove.

As the aspect ratio of the groove increases, the required process time to fill the resist into the mold groove increases because it becomes difficult to flow into the narrow groove by viscous resistance. Also, the filling factor increases linearly as the process time proceeds.

The required process time to fill the groove by the resist is simulated for various aspect ratios of the mold grooves as shown in Fig.9. As the aspect ratio increases, the required process time increases because the resist is hardly flow into the vertical direction in the narrow groove. On the other hand, it takes longer time to flow lateral direction in lower aspect ratio of the groove. As a result, the required process time has local minimum when the aspect ratio is around 0.2 , which will be modulated by the resist initial thickness or viscosity.

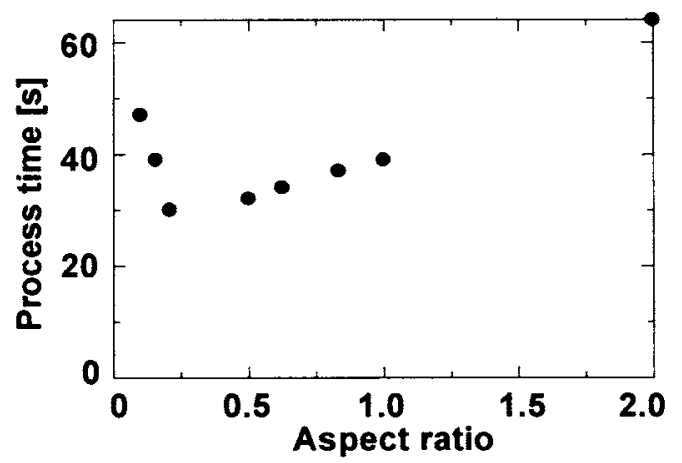

Figure 9. Time evolutions of the filling factor in various aspect ratio of the groove.

\section{Conclusion}

Time evolutions of the resist deformation process are investigated by numerical simulation and experimental study. The resist is lifted up at the beginning of the deformation process and gradually deformed as the time proceeds. The time evolution of the resist profiles are relatively good agreement with each other in qualitatively. Also, the required time to fill the resist into the groove is investigated. As the aspect ration increases, it takes more time by fluid resistance. On the other hand, long process time is required for low aspect ratio groove for lateral direction flow. There exists specific aspect ratio to mineralize the process time.

\section{Acknowledgements}

A part of this work is supported by NEDO.

\section{References}

[1] S. Y. Chou, P. R. Krauss, and P. J. Renstrom, Appl. Phys. Lett. 67, (1995) 3114.

[2] T. Ohta, M. Hennessey, D. Strand, D. Jablonski, B. Walton and B. Clark, Abstract of the $4^{\text {th }}$ International Nanoimprint and Nanoprint Technology Conference (Nara, 2005) $21 \mathrm{~A}-10$.

[3] Y. Nakada, K. Ninomiya and Y. Takaki, ibid $21 \mathrm{~A}-6-4$.

[4] Y. Hirai, T. Konishi, T. Yoshikawa, and S. Yoshida, J. Vac. Sci. Technol. B 22 (2004)3288.

[5] Y. Hirai, T. Yoshikawa, N. Takagi, S. Yoshida, K. Yamamoto, J. Photopolym. Sci. Technol., 16, (2003) 615 . 